\title{
FULL PAPER
}

\section{Phase-contrast and magnification radiography at diagnostic X-ray energies using a pseudo-microfocus $X$-ray source}

\author{
${ }^{1} \mathrm{C} J$ KOTRE, PhD and ${ }^{2} \mathrm{~K} J$ ROBSON, PhD \\ ${ }^{1}$ Christie Medical Physics and Engineering, The Christie NHS Foundation Trust, Manchester, UK \\ ${ }^{2}$ Regional Medical Physics Department, Newcastle-upon-Tyne Hospitals NHS Foundation Trust, Newcastle-upon-Tyne, UK \\ Address for correspondence: Dr Colin John Kotre \\ E-mail: john.kotre@christie.nhs.uk
}

Objective: To investigate the use of conventional diagnostic $\mathrm{X}$-ray tubes for applications in which specialist microfocus sources are normally required.

Methods: A conventional diagnostic X-ray tube was used in conjunction with a range of apertures to investigate improvements in spatial resolution using a line-pairs test object. Phase-contrast effects were investigated by varying source-to-object and object-to-receptor distances using a 2-French catheter as a clinically realistic test object.

Results: For magnification radiography using a computed radiography receptor and conventional $X$-ray tube with a 1-mm nominal focus size, the limiting spatial resolution was improved from 3.55 line-pairs per millimetre, for a conventional contact image, to 5.6 line-pairs per millimetre, for a $\times 2$ magnified view with a $250-\mu \mathrm{m}$ aperture. For inline phase-contrast radiography, phase contrast enhancement of a 2-French catheter was demonstrated, and the expected trends with variations in source-toobject and object-to-receptor distances were found. Images of a neonatal phantom demonstrated a subtle improvement in visibility of a superimposed 1-French catheter simulating a percutaneously inserted central catheter for no increase in patient radiation dose.

Conclusion: Spatial resolution improvement and visible phase contrast can be produced in clinically relevant objects using a pseudo-microfocus geometry at X-ray energies in the normal diagnostic range, using conventional diagnostic $X$-ray tubes and image receptors. The disadvantages of the proposal are the large distances required to produce phase contrast and limitations imposed by the resulting tube loading.

Advances in knowledge: It is possible to use conventional diagnostic $X$-ray equipment in applications that normally require microfocus $\mathrm{X}$-ray sources. This presents some possibilities for clinical applications.
The rotating anode X-ray tube is by far the most common $\mathrm{X}$-ray source used in diagnostic radiology. The engineering compromises inherent in the bevelled tungsten-rhenium rotating anode design deliver a relatively high radiation output with an adequate field coverage and heat dissipation for most radiology work, combined with an X-ray focus size that does not lead to visible geometric unsharpness in normal use. A common nominal X-ray focus size for general radiography is 1.0 or $1.2 \mathrm{~mm}$ with a switchable $0.6-\mathrm{mm}$ fine focus. Although these sizes cover normal medical radiographic imaging requirements, there are specialist applications where a microfocus $\mathrm{X}$-ray source is required. Commercial laboratory microfocus X-ray units commonly operate at relatively low energies and at low output, the output being restricted by the heat capacity of the typically stationary anode. The objective of this article is to examine the possibility of using the standard diagnostic
$\mathrm{X}$-ray tube in conjunction with a small aperture to act as a pseudo-microfocus source. In particular, the possibilities for high geometric magnification radiography and phasecontrast radiography are experimentally demonstrated.

\section{PSEUDO-MICROFOCUS X-RAY GEOMETRY}

The proposed pseudo-microfocus X-ray source consists simply of a standard diagnostic X-ray tube, to which is added an aperture of the required microfocus diameter. A diagrammatic representation of the geometry is shown in Figure 1. The term pseudo-microfocus has been adopted to distinguish this proposal from the conventional microfocus $\mathrm{X}$-ray tube, where the focus is actually on the tube anode. The geometry is identical to that used in pinhole radiography, a common quality control test used to ascertain the dimensions of the X-ray focus. The result of a conventional pinhole radiograph, with the aperture typically $30 \mathrm{~cm}$ from 
the focus, is a magnified image of the tube focus from which its dimensions can be measured. The practical variation explored in this article is to place the aperture much closer to the tube focus in order to increase the size of the focal spot image and to use it as a field in which to image small objects. From Figure 1, the usable field size at the image receptor, $h$, is related to the size of the tube focus, assumed for simplicity to be a square of side $f$, the distance from the tube focus to the aperture, $g$, and the distance from the aperture to the receptor, $d$, as:

$$
h=\frac{f d}{g}
$$

so for maximum usable field size in this geometry, the largest nominal focus size is required, together with the smallest practicable distance, $g$. With $g$ small, it would be expected that the radiation output (e.g. $\mu \mathrm{Gy} \mathrm{mAs}{ }^{-1}$ ) would be approximately related to the relative areas of the tube focus and the aperture. The radiation output would therefore be expected to be proportional to the area of the pseudo-microfocus, but since the limitations of local anode heating impose the same restriction on conventional $\mathrm{X}$-ray tubes, ${ }^{1}$ this does not represent a specific disadvantage of the pseudo-microfocus arrangement, and since the choice of aperture is variable, the trade-off between output, pseudofocus size and tube loading can be optimized.

In order to use the field size, $h$, for useful projection imaging, it is necessary to correct for the inhomogeneity of the projected beam caused by the non-uniformity of X-ray production at the tube target. For a digital image receptor, the flat-fielding correction required can be made by logarithmic subtraction of the image containing the object and a previously stored image of the non-uniform field without the object. This divides the pixel values in the object image by those in the correction image to suppress the beam inhomogeneity. The effectiveness of this correction will depend to some extent on the diameter of the aperture (i.e. the pseudo-microfocus size), as very small apertures will produce a sharp well-modulated image of the tube focus, which is relatively difficult to correct for, whereas larger aperture sizes produce a more blurred and less-modulated image of the tube focus, which is more easily corrected and less sensitive to misregistration of the correction image. This effect is demonstrated in the results below.

\section{EXPERIMENTAL MEASUREMENTS}

All experiments were carried out on a Siemens Opti 150/30/50C $\mathrm{X}$-ray tube (Siemens Healthcare, Erlangen, Germany) with nominal 1- and 0.6-mm foci. Four test apertures were used, with the dimensions of each measured using a microscope with graticule mounted on a numerically controlled milling machine capable of a positioning accuracy of $\pm 5 \mu \mathrm{m}$. In order to mount the test apertures as close to the tube focus as possible, the light beam diaphragm assembly was removed for the duration of the experiment. All images were recorded on an Agfa ${ }^{\mathrm{TM}}$ ADCC-HR (Agfa HealthCare, Mortsel, Belgium) computed radiography (CR) system for which the signal transfer property had been previously measured $^{2}$ and a linearization correction fitted using the method used by Mackenzie. ${ }^{3}$ The CR plates used were $24 \times 30 \mathrm{~cm}$ and had a pixel pitch of $0.11 \mathrm{~mm}$.

\section{Radiation output}

To enable appropriate exposure factors to be set for the imaging experiments, the air kerma at a distance, $d$, of $2.5 \mathrm{~m}$ was measured with a $\operatorname{Radcal}^{\circledR} 6 \mathrm{cc}$ ionization chamber (Radcal Corporation, Monrovia, CA) without an aperture and then with each of four test apertures. The satisfactory alignment of the ionization chamber and radiation field was confirmed in each case by using an X-ray image intensifier to view the positioning. Output measurements were performed at the standard tube potential of $81 \mathrm{kVp}$ often used for quality control measurements.

\section{Magnification radiography}

As a simple test of the proposed pseudo-microfocus geometry, images of a Hüttner type 25a line-pairs test object were produced using aperture sizes of a nominal 60 and $250 \mu \mathrm{m}$, with a geometrical magnification in the range 1.5-2. In each case, a correction image identical to the test image but without the test object in place was recorded. For comparison, conventional contact and magnification images with no aperture were also made using the 1-mm focus.

Figure 1. Diagrammatic representation of the pseudo-microfocus imaging geometry showing the relationship of the quantities defined in Equations (1) and (2). The distances focal spot size ( $f$ ) and focus to aperture distance $(g)$ are shown much enlarged for clarity. $d$, aperture to receptor distance; $d_{1}$, aperture to object distance; $d_{2}$, object to receptor distance; $h$, useable field size.

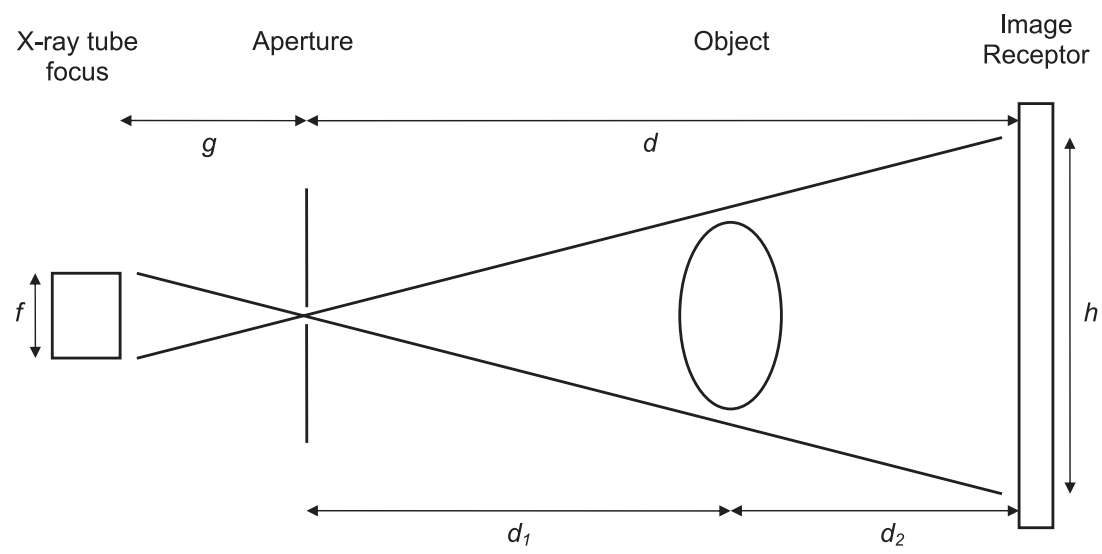




\section{Phase-contrast imaging}

As a spatially coherent X-ray beam propagates through a medium, the phase of the incident wavefront becomes modified in a manner related to the electron density of the medium. The resulting phase gradient across the wavefront is equivalent to a change in direction of the propagation of the wave. The angular deflections from the initial direction of propagation are small but are most pronounced in regions of the object where the X-ray refractive index is varying rapidly, such as at the interface between two different materials. The direction of the deflection will vary from point to point within a general object, depending on the structures present, but will produce a net effect of edge enhancement between structures of differing X-ray refractive index when imaged using an appropriate geometry. A suitable simple geometry consists of a polychromatic microfocus $\mathrm{X}$-ray source, with a distance, $d_{1}$, between the source and the object, and a distance, $d_{2}$, between the object and the image receptor. This geometry is essentially identical to the experimental arrangement used above (Figure 1), but the distances $d_{1}$ and $d_{2}$ are now chosen to produce phase contrast as well as geometrical magnification. The amount of phase contrast produced will depend on the degree of coherence of the polychromatic beam, which can be measured as the lateral coherence length, $L$, given by

$$
L=\frac{\lambda d_{1}}{f}
$$

where $\lambda$ is the wavelength. ${ }^{4}$ As pointed out by Wu and Liu, ${ }^{5}$ this quantity alone does not characterize the phase contrast, as the distance $d_{2}$, the spatial frequency structural component of the object and the spatial frequency response of the receptor are also factors. The requirement for a very small focal spot size is, however, maintained. $\mathrm{Wu}$ and $\mathrm{Liu}^{6}$ discuss the detailed requirements of a microfocus X-ray source suitable for phase-contrast mammography, describing the limitations of the X-ray source as the "key technical bottleneck of developing a clinically feasible phase-contrast X-ray imaging system". The recent review of phasecontrast imaging by Bravin et $\mathrm{al}^{7}$ also discusses X-ray sources.

To demonstrate the suitability of the pseudo-microfocus X-ray source for phase-contrast imaging, a 3-mm perspex edge, a 2-French catheter $(0.67-\mathrm{mm}$ diameter $)$ and a 5 -French catheter (1.67-mm diameter) were imaged using a $250-\mu \mathrm{m}$ aperture under a range of conditions for $d_{1}$ and $d_{2}$. When $d_{1}$ is small, the degree of lateral coherence in the beam will be small and phasecontrast effects will be minimal. Similarly, when $d_{2}$ is small, there will be no distance for phase-contrast effects to be expressed before being recorded at the image receptor. ${ }^{8}$ For larger $d_{1}$ and $d_{2}$, the tube loading becomes very high, and for increasing $d_{2}$, consideration must be made of geometrical blurring and increasing radiation dose at the object if the receptor dose is kept constant. To demonstrate these trends, a set of images were produced for $d_{1}$ fixed at $2 \mathrm{~m}$ in conjunction with $d_{2}=0,1,1.5,2$ and $2.5 \mathrm{~m}$. A second set was recorded with $d_{2}$ fixed at $1 \mathrm{~m}$, and $d_{1}=2,2.5,3$ and $3.5 \mathrm{~m}$. In each case, a correction image identical to the test image but without the test object in place was also recorded. All images were taken at a tube potential of $60 \mathrm{kVp}$ and a receptor dose of approximately $3 \mu \mathrm{Gy}$.

In order to put the results in the context of a possible clinical application, the situation of a 1-French (0.3-mm diameter) percutaneously introduced central catheter in the large vessels surrounding the heart of a neonate was approximately simulated. Such small catheters are often difficult to visualize on radiographs, and accurate positioning is critical. ${ }^{9}$ An anthropomorphic neonatal phantom (ATOM Ltd., Riga, Latvia) ${ }^{10}$ with a 1-French catheter taped to its surface was imaged in a conventional geometry with a focus-to-receptor distance of $90 \mathrm{~cm}$, then in a phase-contrast geometry with $d_{1}=2.5 \mathrm{~m}$ and $d_{2}=2.5 \mathrm{~m}$. Clinically realistic exposure factors of $60 \mathrm{kVp}$ and $2 \mathrm{mAs}$ at a focus-skin distance of $80 \mathrm{~cm}$ were used for the conventional geometry, resulting in a measured entrance skin dose of $87 \mu \mathrm{Gy}$. In the phase-contrast geometry, $60 \mathrm{kVp}$ was maintained and the mAs scaled up to give a measured entrance surface dose of $91 \mu \mathrm{Gy}$ at the much greater focus to skin distance. The same CR plate was used in each case.

\section{RESULTS}

Radiation output

Figure 2 shows the relationship between the output of the pseudo-microfocus source, given conventionally as $\mu \mathrm{Gy}$ per $\mathrm{mAs}$ at $1 \mathrm{~m}$, plotted against the area of the aperture for four aperture sizes. The measured aperture diameters were $65 \mu \mathrm{m}$ $( \pm 5 \mu \mathrm{m}), 100 \mu \mathrm{m}( \pm 5 \mu \mathrm{m}), 250 \mu \mathrm{m}( \pm 5 \mu \mathrm{m})$ and $365 \mu \mathrm{m}$ $( \pm 5 \mu \mathrm{m})$, and the measured size of the tube focus was $1.35 \times 1.02 \mathrm{~mm}( \pm 0.02 \mathrm{~mm})$. Figure 2 confirms the expected relationship.

\section{Geometric magnification}

Figure 3 shows images of the Hüttner line-pairs object under various conditions of aperture size and magnification. For ease of comparison of image resolution, the images are scaled to the same size. Figure 3a shows a contact image of the test object taken with the conventional nominal $1 \mathrm{~mm}$ focal spot and no aperture. This represents the maximum spatial resolution possible using a conventional radiographic geometry and demonstrates the resolution of the image receptor. The line-pairs group

Figure 2. Measured radiation output in $\mu \mathrm{GymAs}^{-1}$ plotted against the area of an aperture placed close to the X-ray tube focus.

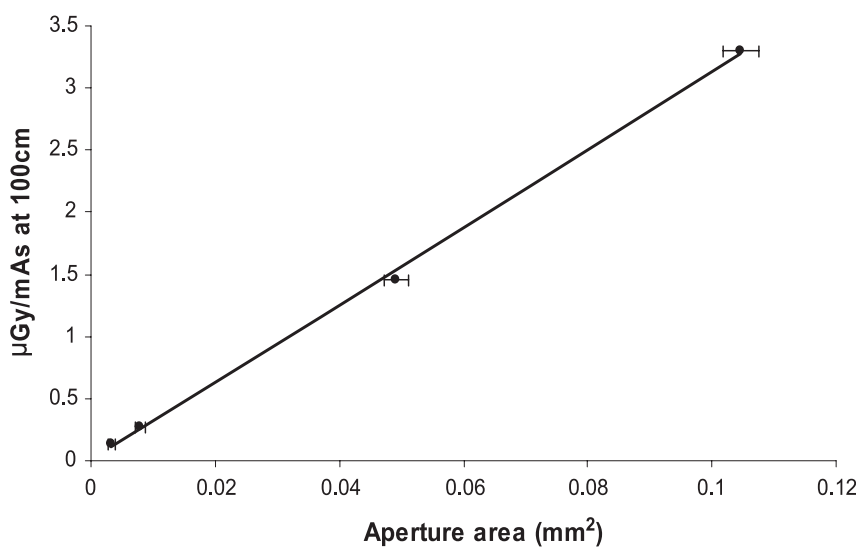


corresponding to 3.55 line-pairs per millimetre can just be resolved. Figure $3 \mathrm{~b}$ shows a geometric magnification of $\times 1.5$, again with the conventional geometry. Geometric blurring is immediately evident and no line-pairs groups can be resolved. Figure $3 \mathrm{c}$ shows a $\times 1.5$ magnified image using the $65-\mu \mathrm{m}$ diameter pseudo-microfocus, in which the 5 line-pairs per millimetre group can be just resolved, but the non-uniformity in the background noise demonstrates the limitations in flatness correction associated with the use of such small apertures. In this image, the resolution is still limited by the receptor pixel pitch of $0.11 \mathrm{~mm}$ rather than by geometrical unsharpness. Figure $3 \mathrm{~d}$ shows a $\times 1.5$ magnified image now with a $250-\mu \mathrm{m}$ diameter pseudo-microfocus, showing 4.5 line-pairs per millimetre, and Figure $3 \mathrm{e}$ shows a $\times 2$ magnified view with the $250-\mu \mathrm{m}$ diameter pseudo-microfocus, showing 5.6 line-pairs per millimetre. In Figure 2 d,e, the improved image uniformity associated with the use of the larger aperture can be seen.

Figure 4 illustrates the effect of aperture diameter on the modulation of the correction image and hence on the difficulty of producing a uniform flat-fielded result. Figure 4a shows a correction image (no object) for the $65-\mu \mathrm{m}$ diameter aperture, and Figure $4 \mathrm{~b}$ shows the corresponding image for the $250-\mu \mathrm{m}$ diameter aperture, showing the expected improvement in field uniformity.

\section{Phase-contrast imaging}

Figure 5 shows a comparison of images of the edge of a 3-mm thick Perspex plate (top), 2-French catheter (middle) and 5-French catheter (bottom) recorded as a contact radiograph, $d_{1}=2 \mathrm{~m}$ and $d_{2}=0 \mathrm{~m}$ (Figure $5 \mathrm{a}$ ), and an image in the phase-contrast geometry with a $250-\mu \mathrm{m}$ diameter pseudo-microfocus, $d_{1}=2 \mathrm{~m}$ and $d_{2}=2.5 \mathrm{~m}$ (Figure $5 \mathrm{~b}$ ). The images demonstrate the edge enhancement and contrast improvement produced by phase contrast superimposed on the conventional attenuation contrast, which gives an overshoot at the boundaries of these objects, which is perceived as better resolution. For direct comparison, the images are shown the same size, but Figure $5 \mathrm{~b}$ also has geometric magnification of $\times 2.25$.

The trends in the amount of phase-contrast enhancement recorded for the 2-French catheter, as the distances $d_{1}$ and $d_{2}$ are altered, are summarized as profile plots in Figures 6 and 7 . Figure 6 shows a pixel-value profile plot for a conventional contact radiograph of the 2-French catheter $\left(d_{1}=2 \mathrm{~m}\right.$, $d_{2}=0 \mathrm{~m}$ ), which can be compared with plots for $d_{1}=2 \mathrm{~m}$ and $d_{2}=1 \mathrm{~m} ; d_{1}=2.5 \mathrm{~m}$ and $d_{2}=1 \mathrm{~m} ; d_{1}=3 \mathrm{~m}$ and $d_{2}=1 \mathrm{~m}$; and $d_{1}=3.5 \mathrm{~m}$ and $d_{2}=1 \mathrm{~m}$. A gradual increase in the overshoots either side of the catheter indicative of phase contrast is seen as the $d_{1}$ distance is increased, which increases the amount of lateral coherence in the beam according to Equation (2). In Figure 7, the phase contrast can be seen to be increasing more rapidly with increasing distance $d_{2}(0,1,1.5,2$ and $2.5 \mathrm{~m})$ for a constant distance $d_{1}$ of $2 \mathrm{~m}$.

Figure 8 shows the comparison of neonatal phantom images with 1-French $(0.3 \mathrm{~mm}$ diameter $)$ catheter taped to its anterior surface to simulate the position of a percutaneously introduced central catheter. Figure $8 \mathrm{a}$ is the conventional contact image and Figure $8 \mathrm{~b}$ the phase-contrast image. Although the catheter can be distinguished in both images, the full length of it is better

Figure 3. Images of the Hüttner line-pairs object under various conditions of aperture size and magnification. For ease of comparison of image resolution, the images are scaled to the same size. (a) Contact image of the test object taken with the conventional nominal 1-mm focal spot and no aperture. (b) Geometric magnification of $\times 1.5$, again with the conventional geometry. (c) $\times 1.5$ magnified image using the $65-\mu \mathrm{m}$ diameter pseudo-microfocus. (d) $\times 1.5$ magnified image with a $250-\mu \mathrm{m}$ diameter pseudomicrofocus. (e) $\times 2$ magnified view with the $250-\mu$ m diameter pseudo-microfocus.

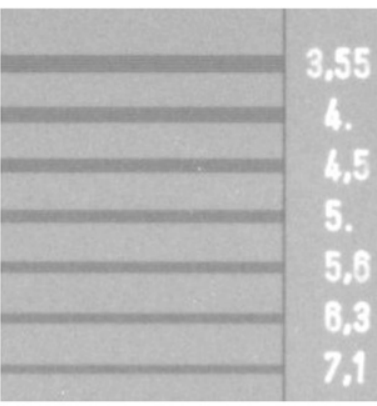

(a)

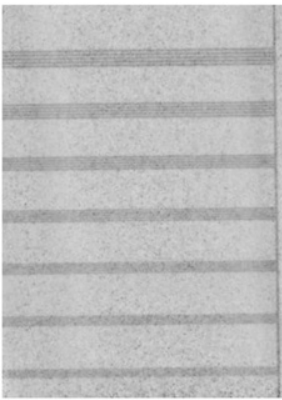

(c)

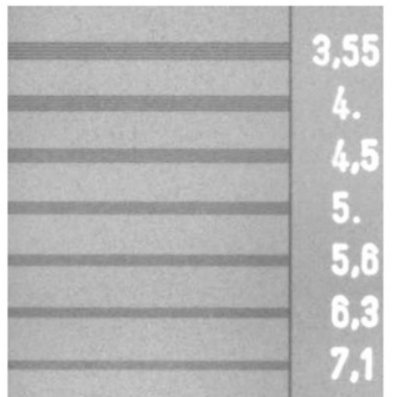

(d)

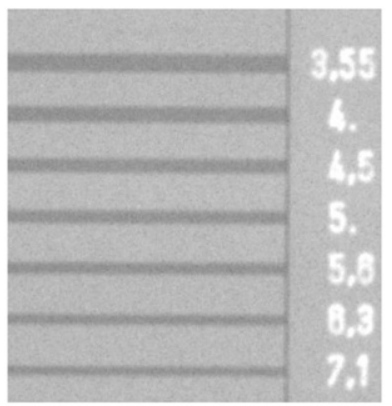

(b)

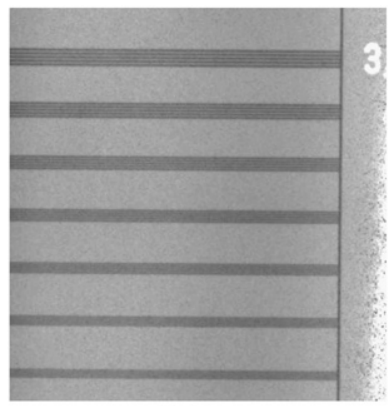

(e) 
Figure 4. The effect of aperture diameter on the modulation of the correction image. (a) A correction image (no object) for the 65- $\mu \mathrm{m}$ diameter aperture and (b) the corresponding image for the 250- $\mathrm{mm}$ diameter aperture, showing the expected improvement in field uniformity.

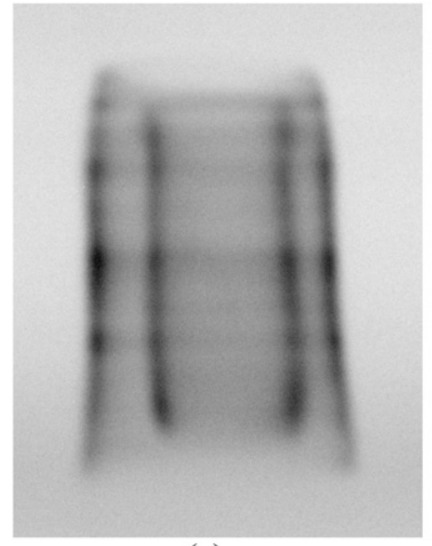

(a)

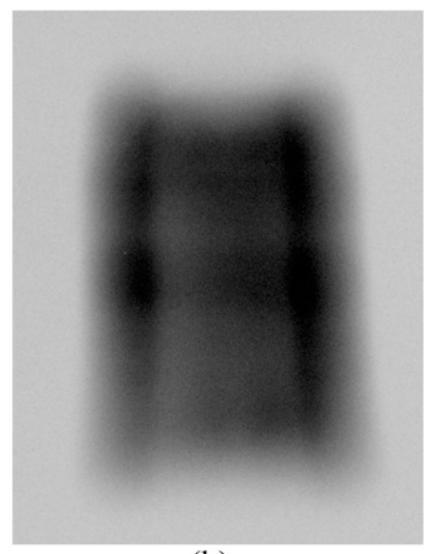

(b)

seen in the phase-contrast image, and features of the phantom, for example, ribs and lung boundaries, are also subtly enhanced.

\section{DISCUSSION}

Patient entrance dose and signal-to-noise ratio Although the improvement in the visibility of certain features owing to the presence of phase contrast is theoretically free of radiation dose penalty, the approach of keeping the receptor dose constant in most of the experiments will have increased the entrance surface dose at the position of the test phantoms owing to the distance from the object-to-image receptor. This approach was taken experimentally for the results shown above in Figures $3,5,6$ and 7 since the performance of computed radiography is limited at low receptor dose by structure noise in the detector. If a more efficient receptor, such as caesium iodide (CsI) phosphor digital radiography, was used, then it would be easier to maintain a similar patient entrance surface dose to conventional

Figure 5. A comparison of images of the edge of a 3-mm thick perspex plate (top), 2-French catheter (middle) and 5-French catheter (bottom) recorded as a contact radiograph, $d_{1}=2 \mathrm{~m}$ and $d_{2}=0 \mathrm{~m}$ (a) and an image in the phase-contrast geometry with a 250- $\mu \mathrm{m}$ diameter pseudo-microfocus, $d_{1}=2 \mathrm{~m}$ and $d_{2}=2.5 \mathrm{~m}$ (b). For direct comparison, the images are shown as the same size, but Figure $4 \mathrm{~b}$ also has geometric magnification of $\times 2.25$.

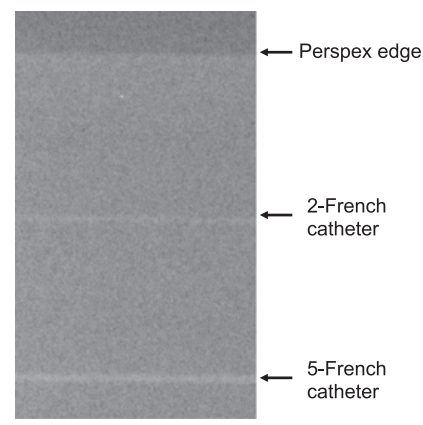

(a)

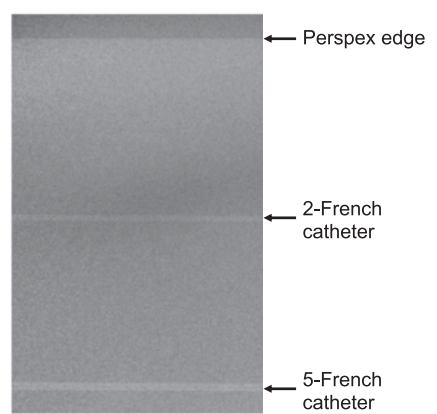

(b)
Figure 6. Pixel-value profiles for a conventional contact radiograph of a 2 -French catheter $\left(d_{1}=2 \mathrm{~m}, d_{2}=0 \mathrm{~m}\right)$, which can be compared with plots for $d_{1}=2 \mathrm{~m}$ and $d_{2}=1 \mathrm{~m} ; d_{1}=2.5 \mathrm{~m}$ and $d_{2}=1 \mathrm{~m} ; d_{1}=3 \mathrm{~m}$ and $d_{2}=1 \mathrm{~m} ;$ and $d_{1}=3.5 \mathrm{~m}$ and $d_{2}=1 \mathrm{~m}$. The overshoots are characteristic of phase contrast.

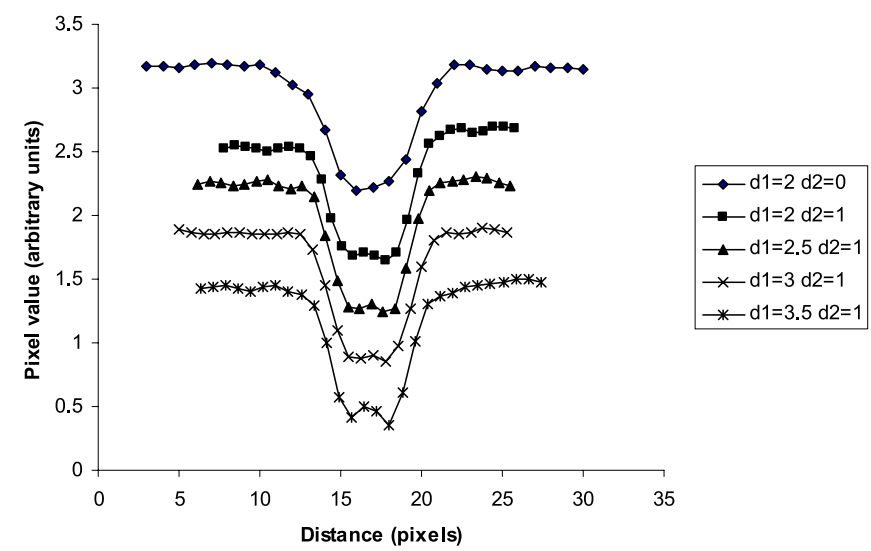

imaging, by allowing the receptor dose to fall, although there would still be a trade-off between the resulting increased quantum noise and the additional information provided by phase contrast. This approach has been used for the results shown in Figure 8, which shows a direct comparison of images in the conventional and phase-contrast geometries at the same patient entrance dose.

A further consideration is the additional noise from the flatfielding step, where quantum noise from the correction image will be added. This can be mitigated by smoothing of the correction image before it is applied. This is particularly effective with moderate aperture sizes where the modulation of the correction image is reduced and principally composed of lowfrequency information (Figure $4 \mathrm{~b}$ ). Another approach would be to use a higher exposure for the correction image, as this will not affect the patient dose.

\section{X-ray tube loading and exposure time}

From Equation (1), it can be seen that, counter intuitively, an improvement to the pseudo-microfocus arrangement can be made by using a larger X-ray tube focus. This produces not only

Figure 7. Pixel-value profiles for the 2-French catheter with the phase contrast increasing more rapidly with increasing distance $d_{2}(0,1,1.5,2$ and $2.5 \mathrm{~m})$ for a constant distance $d_{1}$ of $2 \mathrm{~m}$.

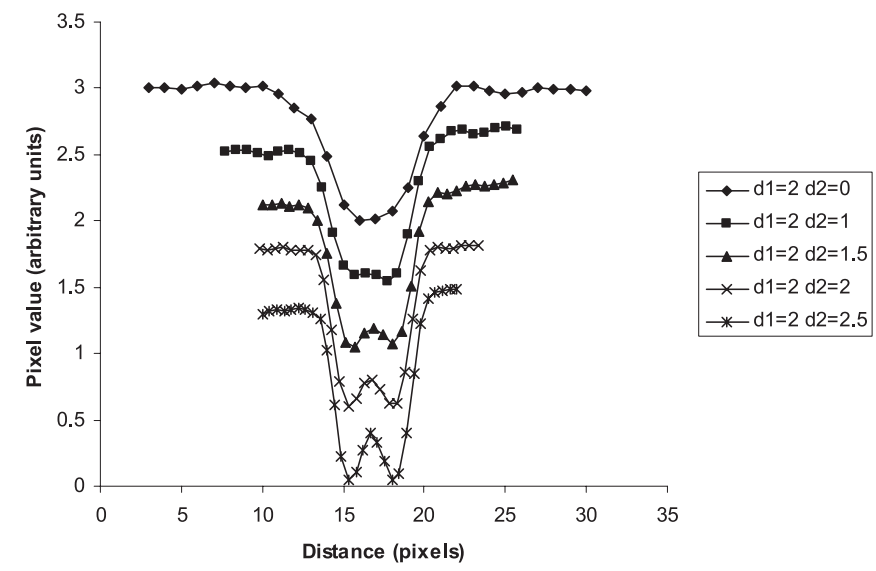


Figure 8. Comparison of images of a neonatal phantom with 1-French catheter taped to its anterior surface to simulate the position of a percutaneously introduced central catheter. (a) The conventional contact image and (b) the phase-contrast image, showing enhancement of the catheter, rib edges and lung boundaries. The skin entrance dose is the same for both images, and the same CR plate was used. (a)

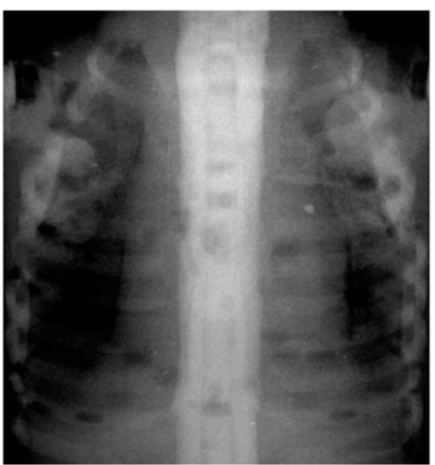

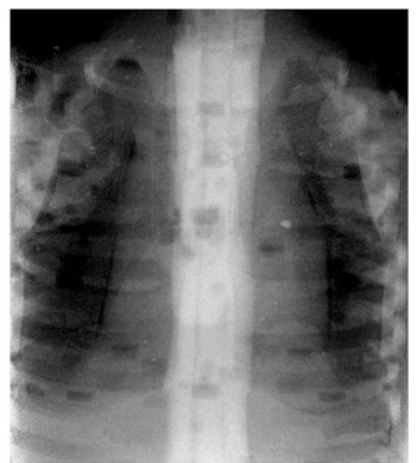

(b) a larger and more practicable usable field size but also increases the heat capacity of the anode, which is one of the limiting factors of the proposal.

\section{Focus-to-image receptor distance}

The use of an inline phase-contrast geometry requires both an adequate combination of small focus size and large focus-object distance to produce an adequate degree of lateral coherhence, ${ }^{4}$ and a large object-receptor distance to allow phase variations to be recorded. ${ }^{8}$ Although this work has demonstrated some imaging advantages to this geometry, it is clear that it would be restricted to projections where the distances could be accommodated.

\section{Other imaging geometry variations}

The phase-contrast geometry demonstrated above aims to add phase contrast as an enhancement to the conventional attenuation image. An alternative approach is to attempt to recover phase information only, and this has been explored using the division of images with different sized microfoci. ${ }^{11}$ This could also be implemented using two different sized pseudomicrofoci, and the image division step could also serve to act as the uniformity correction. The method has the disadvantage that strongly attenuating objects will also display edge enhancement owing to the resulting difference in geometric unsharpness, but in weakly attenuating objects, the phase contrast dominates.

\section{CONCLUSIONS}

It has been demonstrated that a small diameter aperture placed close to the focus of a conventional diagnostic X-ray tube can be made to act as a pseudo-microfocus, provided that appropriate flat fielding of the resulting digital image is carried out. This pseudo-microfocus can be used to produce magnified images without introducing excessive geometric unsharpness, although with a restricted field of view [Equation (1)], and is a suitable source for phase-contrast radiography, also with a restricted field of view. Distance variations of the proposed phase-contrast geometry have been investigated, and it has been shown that visible phase-contrast enhancement can be produced in clinically relevant objects in this configuration at X-ray energies in the normal diagnostic range using conventional diagnostic $\mathrm{X}$-ray tube and image receptor technology.

\section{FUNDING}

Work carried out under NHS contract.

\section{REFERENCES}

1. Dendy PP, Heaton B. Physics for Diagnostic Radiology. 2nd edn. Bristol, UK: Institute of Physics Publishing; 1999.

2. IPEM. Measurement of the Performance Characteristics of Diagnostic X-Ray Systems: Digital Imaging Systems. Institute of Physics and Engineering in Medicine Report 32 Part VII. York, UK: IPEM; 2010.

3. Mackenzie A. Validation of correction methods for the non-linear response of digital radiography systems. Br J Radiol 2008; 81: 341-5. doi: 10.1259/bjr/ 57141560

4. Wilkins SW, Gureyev TE, Gao D, Pogany A, Stevenson AW. Phase-contrast imaging using polychromatic hard x-rays. Nature 1996; 384 335-8.

5. Wu X, Liu H. Clarification of aspects in inline phase-sensitive X-ray imaging. Med Phys 2007; 34: 737-43.

6. Wu X, Liu H. Clinical implementation of X-ray phase-contrast imaging: theoretical foundations and design considerations. Med Phys 2003; 30: 2169-79.

7. Bravin A, Coan P, Suortti P. X-ray phasecontrast imaging: from pre-clinical applications towards clinics. Phys Med Biol 2013; 58: R1-35. doi: 10.1088/0031-9155/ $58 / 1 / R 1$
8. Kotre CJ, Birch IP. Phase contrast enhancement of X-ray mammography: a design study. Phys Med Biol 1999; 44: 2853-66.

9. Nadroo AM, Lin J, Green RS, Magid MS, Holzman IR. Death as a complication of peripherally inserted central catheters in neonates. $J$ Pediatr 2001; 138: 599-601. doi: 10.1067/ mpd.2001.111823

10. Varchenya V, Gubatova D, Sidorin V, Kalnitsky S. Childrens' heterogeneous phantoms and their application in roentgenology. Radiat Prot Dosimetry 1993; 49: 77-8.

11. Donnelly EF, Price RR, Pickens DR. Dual focalspot imaging for phase extraction in phasecontrast radiography. Med Phys 2003; 30: 2292-6. 